\title{
AN INVARIANT FOR ALMOST-CLOSED MANIFOLDS
}

\author{
BY DAVID L. FRANK ${ }^{1}$ \\ Communicated by S. Smale, November 21, 1967
}

1. Let $M^{n}$ be a compact, oriented, connected, $n$-dimensional differential manifold with $\partial M$ (boundary $M$ ) homeomorphic to the $n-1$ sphere $S^{n-1}$. Then $\partial M$ represents an element $[\partial M]$ of $\Gamma^{n-1}$, the group of differential structures (up to equivalence) on $S^{n-1}$. We consider the (much studied) problem of expressing $[\partial M]$ in terms of "computable" invariants of $M$.

Let $\pi_{n-1}$ be the $n-1$ stem, $J_{0}: \pi_{n}(\mathrm{BSO}) \rightarrow \pi_{n-1}$ the classical $J$-homomorphism, and $\pi_{n-1}^{\prime}$ the cokernel of $J_{0}$. In [5], a map $P: \Gamma^{n-1} \rightarrow \pi_{n-1}^{\prime}$ was defined (see below). We will define an invariant $\Delta(M)$ which is a subset of $\pi_{n-1}^{\prime}$ (and of ten consists of a single element). The main theorem states: $P[\partial M] \in \Delta(M)$.

In a strong sense, the definition of $\Delta(M)$ involves only homotopy theory. Moreover, $\Delta(M)$ seems amenable to computation by standard techniques of algebraic topology. We illustrate this below and, as applications, give explicit examples (1) of a manifold $M^{n}, n$ odd, with $[\partial M] \neq 0$, and (2) of $M^{n}, n$ even, with $[\partial M]$ not only $\neq 0$, but in fact with $[\partial M]$ not even contained in $\Gamma^{n-1}(\partial \pi)$, the subgroup in $\Gamma^{n-1}$ of elements which bound $\pi$-manifolds. (Examples of $M^{n}, n$ even, with $[\partial M] \neq 0$ are of course well known.) Other applications, and detailed proofs, will appear elsewhere.

REMark 1. By [5], kernel $P=\Gamma^{n-1}(\partial \pi)$. If $n$ is odd, $\Gamma^{n-1}(\partial \pi)=0$, so $P$ is injective, while if $n \equiv 2$ (4), kernel $P \subseteq Z_{2}$. If $n \equiv 0$ (4), kernel $P$ tends to be large (but see $\$ 5$ ).

Let BSO, BSPL, BSTop be the stable classifying spaces for orientable vector bundles, piecewise-linear $(=P L)$ bundles, topological bundles. There are maps $J_{G}: \pi_{n}(\mathrm{BSG}) \rightarrow \pi_{n-1}(\mathrm{G}=\mathrm{O}, \mathrm{PL}$, Top) and a commutative diagram with exact rows

$$
\begin{aligned}
& 0 \rightarrow \pi_{n}(\mathrm{BSO}) \stackrel{f}{\rightarrow} \pi_{n} \text { (BSPL) } \stackrel{g}{\rightarrow} \Gamma^{n-1} \rightarrow 0
\end{aligned}
$$

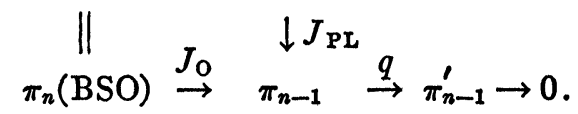

If $z \in \Gamma^{n-1}$, define $P(z)$ as $q\left(J_{\mathrm{PL}}(y)\right)$, where $g(y)=z$.

2. On Thom complexes. Let $\beta$ be an oriented (topological) $k$-disk bundle over a CW-complex $X, T(\beta)$ the Thom complex. If $X$

1 National Science Foundation Fellow. 
$=Y \cup_{d} e^{n}\left(=Y\right.$ with an $n$-cell attached by $\left.d: \partial e^{n} \rightarrow Y\right)$, then $T(\beta)$ $=T(\beta \mid Y) \cup_{\phi} e^{n+k}$. Also, if $* \in Y^{Y}$ is the basepoint, we have an inclusion $i: S^{k}=T(\beta \mid *) \rightarrow T(\beta \mid Y)$. Assume $k, n \geqq 2$.

Proposition 1. Let $X=Y \cup e^{n}, Y$ a connected $n-1$ dimensional complex. Let $\alpha, \beta$ be oriented (topological) $k$-disk bundles over $X$ with $\alpha \mid Y$ isomorphic to $\beta \mid Y$. Let $T(\beta)=T(\beta \mid Y) \cup_{\phi} e^{n+k}$, $[\phi] \in \pi_{n+k-1}(T(\beta \mid Y))$. Suppose $T(\alpha)$ is reducible [4]. Then $[\phi] \in$ image $i_{*}: \pi_{n+k-1}\left(S^{k}\right) \rightarrow \pi_{n+k-1}(T(\beta \mid Y))$.

REMARK 2 . If $\delta$ is a $k$-disk bundle over $S^{n}$ derived from $(\alpha, \beta)$ by the difference construction, then in fact $[\phi]= \pm i_{*} J(\delta)$, where $J=J_{\text {Top }}: \pi_{n}$ (BSTop) $\rightarrow \pi_{n-1}=\pi_{n+k-1}\left(S^{k}\right)$ (here we assume $k$ is large, although the remark has a nonstable analogue).

Remark 3. Proposition 1 can be generalized to the case in which $T(\alpha)$ is not necessarily reducible. One then has a statement about the difference of the attaching maps in the two Thom complexes.

3. Definition of the invariant. Given $M^{n}$ as in $\$ 1$, let $M^{*}$ be the closed PL manifold $M \cup$ Cone $(\partial M)$. Let $\nu_{M}$ be the $k$-dimensional normal bundle of $M$ in Euclidean $n+k$ space ( $k$ large). Using the fact that the map $\pi_{n-1}$ (BSO) $\rightarrow \pi_{n-1}$ (BSPL) is injective, one sees that $\nu_{M}$ extends to a vector bundle $\nu^{*}$ on $M^{*}$. Let $T\left(\nu^{*}\right)=T\left(\nu_{M}\right) \cup_{\phi} e^{n+k}$, $[\phi] \in \pi_{n+k-1}\left(T\left(\nu_{M}\right)\right)$. Apply Proposition 1 with $\alpha=\nu_{\mathrm{PL}}\left(M^{*}\right)=k$ dimensional PL normal bundle of $M^{*}, \beta=\nu^{*}$. We conclude that $[\phi]$ eimage $i_{*}: \pi_{n-1}=\pi_{n+k-1}\left(S^{k}\right) \rightarrow \pi_{n+k-1}\left(T\left(\nu_{M}\right)\right)$. Define $\Delta^{\prime}\left(\nu^{*}\right) \subseteq \pi_{n-1}$ as $\left\{y \in \pi_{n-1}: i_{*}(y)=[\phi]\right\}$. Let $\Delta\left(\nu^{*}\right)=q\left(\Delta^{\prime}\left(\nu^{*}\right)\right) \subseteq \pi_{n-1}^{\prime}$. Now $\Delta^{\prime}\left(\nu^{*}\right)$ depends on the particular vector bundle extension $\nu^{*}$ of $\nu_{M} ; \Delta\left(\nu^{*}\right)$, however, does not. We may therefore define:

$$
\Delta(M)=\Delta\left(\nu_{M}\right)=\Delta\left(\nu^{*}\right),
$$

where $\nu^{*}$ is any vector bundle on $M^{*}$ extending $\nu_{M}$.

TheOREM 1. Let $M^{n}$ be a compact, oriented, connected, differential $n$-manifold with $\partial M$ homeomorphic to $S^{n-1}$. Then $\pm P[\partial M] \in \Delta(M)$.

Proof (SKETCH). Let $\nu_{\mathrm{PL}}\left(M^{*}\right), \nu^{*}$ be as above. It can be shown that there is a $y \in \pi_{n}(\mathrm{BSPL})$ with $g(y)=[\partial M]$ and such that $y$ is a difference bundle for $\left(\nu_{\mathrm{PL}}\left(M^{*}\right), \nu^{*}\right)$. By Remark $2, T\left(\nu^{*}\right)=T\left(\nu_{M}\right) \cup_{\phi} e^{n+\hbar}$, where $[\phi]= \pm i_{*} J(y)$. But $q(J(y))=P[\partial M]$. Thus $\pm P[\partial M] \in \Delta(M)$.

4. We give some applications of Theorem 1 ( $M$ is always as in $\S 1$ ). Definition. A manifold $M$ is of type $m$ with respect to $(X, \beta)$ if $X$ is a CW-complex with $m$ cells in positive dimensions, $\beta$ is a vector 
bundle over $X$, and there is a map $f: M \rightarrow X$ with $f^{*}(\beta)$ stably isomorphic to $\nu_{M}$.

We consider here manifolds of type one. This class of manifolds is certainly wide enough to be of geometric interest. For example, the following are of type one (with respect to $S^{i}$ and some $\beta \in \pi_{i}$ (BSO)).

(a) $i-1$ connected $M^{n}, n=2 i$.

(b) $i-1$ connected $M^{n}, n=2 i+1, \quad i \neq 1,2$ (8).

(c) The manifolds $M^{n}\left(g_{1}, g_{2}\right)$, where $g_{1} \in \pi_{i-1}(\mathrm{SO}(n-i))$ and $g_{2} \in \pi_{n-i-1}(\mathrm{SO}(i))$, formed by plumbing an $(n-i)$-disk bundle over $S^{i}$ (with characteristic map $g_{1}$ ) and an $i$-disk bundle over $S^{n-i}$ (with characteristic map $g_{2}$ ), provided that the bundle over $S^{n-i}$ is stably trivial.

Suppose $M^{n}$ is of type one with respect to $\left(S^{i}, \beta\right)$, and let $j: S^{n-1} \rightarrow M$ be the inclusion of $\partial M$ into $M$.

Definition. $\Phi_{\beta}(M)=\left\{f j \mid f: M \rightarrow S^{i}\right.$ and $f^{*}(\beta)$ stably isomorphic to $\left.\nu_{M}\right\}$. (Thus $\Phi_{\beta}(M) \subseteq \pi_{n-1}\left(S^{i}\right)$.)

$\Phi_{\beta}$ appears to be an important invariant for the study of manifolds of type one. We take the view that $\Phi_{\beta}(M)$ is "known" or computable. This is certainly reasonable for cases (a), (b), (c) above. For example, in cases (a) or (b) one can usually express $\Phi_{\beta}(M)$ in terms of more standard invariants (Pontryagin classes, behavior of cohomology operations, etc.) and in case (c) we have:

Lemma 1. Let $M^{n}=M\left(g_{1}, g_{2}\right)$ as in (c). Then $M$ is of type one with respect to $\left(S^{i}, g_{1}\right)$, and $J\left(g_{2}\right) \in \Phi_{\theta_{1}}(M)$. (Here $J: \pi_{n-i-1}(\mathrm{SO}(i)) \rightarrow \pi_{n-1}\left(S^{i}\right)$.)

We wish to compute $\Delta(M)$ in terms of $\Phi_{\beta}(M)$.

ThEOREM 2. Let $M^{n}$ be of type one with respect to $\left(S^{i}, \beta\right), \beta \in \pi_{i}$ (BSO). Suppose the composition $x y \in \Phi_{\beta}(M)$, where $y \in \pi_{n-1}\left(S^{p}\right), x \in \pi_{p}\left(S^{i}\right)$, $i<p<n-1$; and suppose $x^{*}(\beta)=0$. Then

(i) The Toda bracket $\left\langle J_{0}(\beta), S_{\beta}(x), S(y)\right\rangle$ is defined.

(ii) $\pm \Delta(M) \subseteq q\left\langle J_{0}(\beta), S_{\beta}(x), S(y)\right\rangle$.

Explanation. Here $S: \pi_{n-1}\left(S^{p}\right) \rightarrow \pi_{n-1-p}$ is the suspension map; $S_{\beta}:\left\{x \in \pi_{p}\left(S^{i}\right): x^{*}(\beta)=0\right\} \rightarrow \pi_{p-i}$ is a certain "twisted" suspension map, which we will not define here.

REMARK. Theorem 2 can be generalized; for example, one may replace $S^{p}$ by an arbitrary complex.

LEMMA 2. For a suitable generator $\gamma$ of $\pi_{4}(\mathrm{BSO}), S_{\gamma}: \pi_{7}\left(S^{4}\right) \rightarrow \pi_{8}$ satisfies: 


$$
\begin{aligned}
S_{\gamma}(B) & =0, B \text { the Hopf map, } \\
S_{\gamma}(t) & =S(t), t \text { an element of finite order. }
\end{aligned}
$$

Recall that $\pi_{8}\left(S^{4}\right)=Z_{2} \oplus Z_{2}=\{c\} \oplus\{d\}$, where (in notation of [9]) $c=E \nu^{\prime} \circ \eta_{7}, d=\nu_{1} \circ \eta_{7}$.

As an illustration of Theorem 2, we have

TheOREM 3. Let $M^{9}$ be of type one with respect to $\left(S^{4}, \gamma\right), \gamma$ as in Lemma 2. Recall $\Gamma^{8}=Z_{2}$. Then

(i) If $\mathrm{O}$ or $d \in \Phi_{\gamma}(M)$, then $[\partial M]=0$.

(ii) If $c$ or $c+d \in \Phi_{\gamma}(M)$, then $[\partial M] \neq 0$.

Proof (Sketch). Suppose that $c \in \Phi_{\gamma}(M)$. By Theorems 1 and 2, $P[\partial M] \in \Delta(M) \subseteq q\left\langle J(\gamma), \quad S_{\gamma}\left(E \nu^{\prime}\right), \quad S\left(\eta_{7}\right)\right\rangle=q\left\langle J(\gamma), S\left(E \nu^{\prime}\right), \quad S\left(\eta_{7}\right)\right\rangle$ (by Lemma 2). Using [9, especially Chapter VI], one calculates that this set is the nonzero element of $\pi_{8}^{\prime}=Z_{2}$. Other cases follow similarly.

Examples. 1 . There is a $z \in \pi_{4}(\mathrm{SO}(4))$ with $J(z)=c$. Consider the 9-manifold $M\left(g_{1}, g_{2}\right)$ with $g_{1} \in \pi_{3}(\mathrm{SO}(5))$ stably equal to $\gamma$ and $g_{2}=z$. By Lemma 1, $J(z)=c \in \Phi_{\gamma}(M)$. By Theorem 3, $[\partial M] \neq 0$.

2. There is a $w \in \pi_{6}(\mathrm{SO}(4))$ with $J(w)=\alpha_{1}(4) \circ \alpha_{1}(7)$ [9, p. 178]. Consider the 11-manifold $M\left(g_{1}, g_{2}\right)$ with $g_{1} \in \pi_{3}(\mathrm{SO}(7))$ stably equal to $\gamma$ and $g_{2}=w$. As above, one sees that $P[\partial M] \in \Delta(M)=q\left\langle\alpha_{1}, \alpha_{1}, \alpha_{1}\right\rangle$ $=q\left(\beta_{1}\right)=$ element of order 3 in $\pi_{10}^{\prime}=Z_{2} \oplus Z_{3}$. Thus [ $\left.\partial M\right]$ is of order 3 in $\Gamma^{10}=Z_{2} \oplus Z_{3}$.

3. There is a $v \in \pi_{11}(\mathrm{SO}(4))$ with $J(v)=E \nu^{\prime} \circ \epsilon_{7}[9$, p. 66]. Consider the 16-manifold $M\left(g_{1}, g_{2}\right)$ with $g_{1} \in \pi_{3}(\mathrm{SO}(12))$ stably equal to $\gamma$ and $g_{2}=v$. One sees that $P[\partial M]=q\langle\nu, 2 \nu, \epsilon\rangle=$ generator of $\pi_{16}^{\prime}=Z_{2}$. Thus $[\partial M] \notin \Gamma^{15}(\partial \pi)$.

REMARK. Let $p$ be an odd prime, and let $n=2 p(p-1)-1$. It is known that the $p$-primary component of $\Gamma^{n-1}=Z_{p}$. Theorem 1 gives good information when applied to the problem of detecting the $p$-primary component of $[\partial M], \operatorname{dim} M=n$. For example, one may show that if the $p$-primary component of $[\partial M] \neq 0$, then $q_{1}(M) \neq 0, q_{1}$ the first $(\bmod p) \mathrm{Wu}$ class. In particular, $M$ can not be $2(p-1)$-connected. It may be conjectured that the generator of the $p$-primary component of $\Gamma^{n-1}$ bounds a manifold of the homotopy type of $S^{t} \bigvee S^{n-t}, t=2(p-1)$. This is true if $p=3$ (see Example 2 above).

5. The case $n=4 k$. Define $r: \Gamma^{n-1} \rightarrow Q / Z$ (rationals $\bmod 1$ ) as follows: given $z \in \Gamma^{n-1}$, choose $y \in \pi_{n}$ (BSPL) with $g(y)=z$. Then put $r(z)=\left(p_{k}(y)\right) / b_{k} \bmod 1$, where $p_{k}$ is the $k$ th rational Pontryagin class and $b_{k}=p_{k}(\gamma), \gamma$ a generator of $\pi_{n}$ (BSO). (By Bott, $b_{k}=a_{k}(2 k-1)$ !, $a_{k}=1$ ( $k$ even) or 2 ( $k$ odd).) 
Define $P^{\prime}$ : kernel $r \rightarrow \pi_{n-1}$ as follows: if $r(z)=0$, there is a (unique) $y$ with $g(y)=z$ and $p_{k}(y)=0$. Put $P^{\prime}(z)=J_{P I}(y)$.

LEMMa 3. The pair $\left(r, P^{\prime}\right)$ is injective, in the sense that if $r(z)=0$, then $P^{\prime}(z)$ is defined, and if $P^{\prime}(z)=0$, then $z=0$.

Proof. Assume $r(z)=0$, and let $y \in \pi_{n}$ (BSPL) satisfy $g(y)=z$, $p_{k}(y)=0$. Then $J_{\mathrm{PL}}(y)=P^{\prime}(z)=0$, by assumption. Thus $p_{k}(y)$ $=J_{\mathrm{PL}}(y)=0$. But this implies $y=0$ (see [2], [3], [8]), so $z=0$.

Now given $M^{n}$, define $s(M)$ by

$$
s(M)=\left[p_{k}\left(\nu^{*}\right)-p_{k}\left(\nu_{\mathrm{PL}}\left(M^{*}\right)\right)\right] / b_{k} \bmod 1,
$$

where $\nu^{*}$ is any vector bundle on $M^{*}$ extending $\nu_{M}$.

If $s(M)=0, \nu^{*}$ may be chosen with $p_{k}\left(\nu^{*}\right)=p_{k}\left(\nu_{\mathrm{PL}}\left(M^{*}\right)\right)$. Then define $\Delta^{\prime}(M)$ as $\left\{x \in \pi_{n-1}: i_{*}(x)=[\phi]\right\}$, where $T\left(\nu^{*}\right)=T\left(\nu_{M}\right) \cup_{\phi} e^{n+k}$ (as in $\S 3$ ).

THEOREM $1^{\prime}$. (i) $s(M)=r[\partial M]$. (ii) If $s(M)=0$, then $\pm P^{\prime}[\partial M]$ $\in \Delta^{\prime}(M)$.

REMARK. The invariant $r$ is closely related to Milnor's $\lambda$ invariant [7]. In fact, $b_{k} \cdot r(z)=\lambda(z), \bmod 1$.

Let $d_{k}$ be the denominator of $B_{k} / 4 k, B_{k}$ the $k$ th Bernoulli number. Let $j_{k}$ be the order of the image of $J_{0}: \pi_{4 k}$ (BSO) $\rightarrow \pi_{4 k-1}$. Recall that $j_{k}=t_{k} d_{k}, t_{k}=1$ or 2 . In every known case, $t_{k}=1$ (for example, $k$ odd [1], $k=2$ or 4 , or $k$ as in [6]).

In the rest of this section, $\operatorname{dim} M=4 k$, where $t_{k}=1$.

THEOREM 4. Let $M$ be a spin manifold, and suppose $\Delta(M)=0$. Then $[\partial M]=0$ if and only if $s(M)=0$ and $\hat{A}\left(M^{*}\right)$, the $\hat{A}$-genus of $M^{*}$, is integral. $^{2}$

Proof. Necessity is well known.

Sufficiency. Let $T\left(\nu^{*}\right)=T\left(\nu_{M}\right) \cup \cup_{\phi} e^{n+k}$, where $p_{k}\left(\nu^{*}\right)=p_{k}\left(\nu_{\mathrm{PL}}\left(M^{*}\right)\right)$. Let $y \in \Delta^{\prime}(M)$; i.e. let $i_{*}(y)=[\phi]$. One may show that $\hat{A}\left(M^{*}\right)=e(y)$ $\bmod 1$, where $e$ is the invariant of [1]. Also, $\Delta(M)=0$ implies $y$ Eimage $J_{0}$. But by [1], $y \in$ image $J_{0}$ and $e(y)=0$ imply $y=0$ (if $\left.t_{k}=1\right)$. Thus $\Delta^{\prime}(M)=0$ and the theorem follows from Theorem $1^{\prime}$ and Lemma 3.

EXample (Kervaire-Milnor). Suppose $\nu_{M}$ is the trivial bundle. Then $[\partial M]=0$ if and only if $\left(p_{k}\left(M^{*}\right)\right) / b_{k}$ and $\hat{A}\left(M^{*}\right)$ are integral.

Proof. One sees that $\Delta(M)=0$ and that $s(M)=\left(p_{k}\left(M^{*}\right)\right) / b_{k} \bmod 1$. Apply Theorem 4. $1 / a_{\mathrm{k}}$.

${ }^{2}$ We use a definition of $\hat{A}$ which differs from the customary one by a factor of 
6. Theorem 1 can be improved somewhat. Let $D\left(\nu_{M}\right)$ be the set of all differential structures on the topological manifold $M$ with normal bundle equal to $\nu_{M}$; by restricting each such structure to $\partial M$, we obtain a subset $\Gamma\left(\nu_{M}\right)$ of $\Gamma^{n-1}$. The argument in the proof of Theorem 1 shows that $\pm P\left(\Gamma\left(\nu_{M}\right)\right) \subseteq \Delta\left(\nu_{M}\right)$. (Using properties of the map $J_{\mathrm{PL}}$ $[2],[3],[8]$, one can sometimes show that this inclusion is an equality.)

\section{REFERENCES}

1. J. F. Adams, On the groups $J(x)$. IV, Topology 5 (1966), 21-71.

2. D. Frank, The piecewise linear J-homomorphism and the smoothing problem, Notices Amer. Math. Soc. 13 (1966), 848.

3. - Reducible Thom complexes and the smoothing problem, $\mathrm{Ph}$. D. Thesis, University of California, Berkeley, Calif., 1967.

4. I. M. James, Spaces associated with Stiefel manifolds, Proc. London Math. Soc. 9 (1959), 115-140.

5. M. Kervaire and J. Millnor, Groups of homotopy spheres, Ann. of Math. 77 (1963), 504-537.

6. M Mahowald, On the order of the image of $J$, Topology 6 (1967), 371-378.

7. J. Milnor, Differential structures on spheres, Amer. J. Math. 81 (1959), 962-972.

8. D. Sullivan, Triangulating homotopy equivalences, mimeo. notes, Warwick, 1966.

9. H. Toda, Composition methods in homotopy groups of spheres, Ann. of Math. Studies 49 (1962).

University of California, Berkeley, aNd Mathematical Institute, OXFord 